\title{
Tendencias en mercadotecnia para posgrados en México
}

\author{
Marketing trends for graduate studies in Mexico
}

\author{
RAMOS, Patricia ${ }^{1}$ \\ BARERA, Alejandra ${ }^{2}$ \\ RAMÍREZ, Flor Rocio ${ }^{3}$
}

\begin{abstract}
Resumen
Se realizó una evaluación de la oferta de posgrados del área de mercadotecnia en universidades de México, que tienen similaridad en sus planes de estudio. El objetivo general lo constituyó la compilación y el análisis de elementos que desarrollan para posicionarse en distintas regiones. Con un abordaje cuantitativo y cualitativo se generó una matriz de particularidades en el ámbito de la mercadotecnia. Como resultados se destacan: ofrecer habilidades para la dirección de las empresas y la investigación de mercados.
\end{abstract}

Palabras clave: mercadotecnia; tendencias; posicionamiento

\begin{abstract}
An evaluation was made of the offer of postgraduate degrees in the marketing area in universities in Mexico, which have similarities in their study plans. The general objective was the compilation and analysis of elements that they develop to position themselves in different regions. With a quantitative and qualitative approach, a matrix of particularities was generated in the field of marketing. As results stand out offering skills for the management of companies and market research.

Key words: marketing; trends; positioning
\end{abstract}

\section{Introducción}

El objetivo de este trabajo es analizar los factores que ofertan las IES (Instituciones de Educación Superior) en México para identificar las tendencias en el ámbito de la mercadotecnia. Debido a que la mercadotecnia sigue siendo importante para cualquier organización y en la actualidad se requieren nuevas metodologías para los desafíos globales.Incluyendo también a la educación superior que como lo comentan Bermeo, Correa, Moya, Arias \& Arango (2020) hoy las exigencias de información se han acrecentado de manera exponencial.

En el mismo sentido Pombosa, Espín, Morales, Moyano \& Burgo (2018) exponen que la competitividad es una necesidad, por lo que la incorporar programas de posgrados de calidad, es necesario y como lo destacan Cueto,

\footnotetext{
${ }^{1}$ Profesor Investigador. Departamento de Ciencias Adminsitrativas. Universidad Autónoma de Ciudad Juárez. México.pramos@uacj.mx

2 Estudiante de Doctorado. El Colegio de Chihuahua. México. vazbar19@gmail.com

${ }^{3}$ Profesor Investigador. Departamento de Ciencias Administrativa. Universidad Autónoma de Ciudad Juárez. México. rocio.ramirez@uacj.mx
} 
Peña, Reyes \& Cobos (2018) debido a las necesidades actuales es necesario reconfigurar y diseñar programas adecuados para cubrir en el país los requerimientos de las empresas, los de la comunidad estudiantil y de los profesionistas.

La formación profesional tiene gran relevancia, ya que impacta en la eficacia de los futuros profesionales. Recientemente en América Latina, en México y Guerrero, las reformas educativas han trabajado en el incremento de la calidad educativa (Muñoz, Avilés, Divicino, \& Ramírez, 2020).Además, como lo expresan Hernández \& López (2011) los estudios de posgrado pueden facilitar a los alumnos los elementos necesarios para la creación de políticas de calidad en diversas disciplinas, encaminadas a transformar los elementos del sistema como la formación de expertos, mejorar las prácticas de las instituciones y sus departamentos, así como modificar sus metodologías de trabajo y evaluar los currículums, entre otras cosas.

\subsection{Revisión teórica}

Es bien sabido por los estudiantes que egresan y que desean seguir estudiando un posgrado afín a su carrera, o en otro campo disciplinar, que seleccionan una institución también por su calidad educativa, prestigio y horarios flexibles (Cueto, Peña, Reyes \& Cobos, 2018).

Por lo tanto, como lo enfatiza Nieto, Ortega \& Turrent (2017) los posgrados en el área de negocios deben responder a las nuevas exigencias y ofertar programas de formación acertados que integren: conocimientos, habilidades transversales, valores y tendencias que impacten en el ámbito empresarial, con la finalidad de que los egresados planteen procedimientos que sean factibles en las empresas en las que se emplean o se desarrollaran.

Moral, Villegas, Landero \& Cornelio (2020) mencionan que la educación se ha transformado y ha prosperado en diversas investigaciones desde sus objetivos hasta sus métodos, destacando recientemente la educación superior y lo que esta implica. Así como Vega, González, González \& Hernández (2018) exponen que se hace necesario que los profesionales se preparen para contribuir a las problemáticas; y en el mismo sentido se requieren programas que formen estudiantes con habilidades para el desarrollo productivo y habilidades en investigación.

Ya que el escenario empresarial demanda las nuevas tendencias debido a la velocidad con que se presentan las necesidades y cambios para el desarrollo de las organizaciones, el área de mercadotecnia es una oportunidad para trabajar las necesidades de los diversos mercados y su satisfacción y no se queda fuera de cambios y la generación de tendencias (Alonso, 2017). Así mismo como lo indican Vega, González, González \& Hernández (2018) el área de mercadotecnia está presente en todas las dimensiones de las ciencias económicas y administrativas.

Vélez \& Sera (2018) enfatizan que "la mercadotecnia rebasa las fronteras nacionales para adentrarse en los marcos de una economía globalizada, lo que implica el empleo de nuevas estrategias de mercadotecnia, que incluyan en la investigación de mercado la multiculturalidad e interculturalidad" p (28).

Por lo anterior, las nuevas tendencias de mercadotecnia del siglo XXI toman fuerza y presentan un abanico de alternativas para que esta disciplina se adecue a las necesidades de las empresas y desarrollen enfoques personalizados (Luque, Lozano \& Quiroz, 2017)

Y hablar hoy de mercadotecnia como lo expresan Rodríguez, Pineda, \& Castro (2020), resulta un tema de considerable interés por los cambios continuos de los múltiples entornos caracterizados por una evolución y las tendencias diferenciadas, donde la mercadotecnia tendrá que satisfacer necesidades desde sus diversas perspectivas y deberá adaptarse a las nuevos desafíos. 


\section{Metodología}

El abordaje metodológico se desarrolló bajo un enfoque cualitativo-cuantitativo y un muestreo por conveniencia, debido a que no se encontró un registro especifico de universidades que oferten posgrados en el área de mercadotecnia que operen en México. Se utilizo la técnica de la bola de nieve a partir de la información obtenida en diversas páginas web. El trabajo de gabinete sirvió de base conceptual y después con el análisis de los factores se derivó la primera tabla con los elementos de los programas de las universidades, tras lo cual se obtuvieron planes de estudio, objetivos, perfil de egresados, elementos y atributos que motivan su esquema de trabajo en México. Como segunda parte se definieron 12 categorías en el perfil de egreso de las universidades para realizar una comparación entre la manera de operar, además de la base de información obtenida en la tabla 1 de la cual se presenta un resumen. En relación con la metodología cuantitativa, se utilizó una muestra aleatoria probabilística para el análisis de redes, ya que, como aseveran Betancur y Murcia (2019), es un método matemático que tiene diferentes objetivos desde el estudio de redes hasta alineaciones conceptuales que acceden a completar estudios cualitativos y facilitar estrategias para evaluar las relaciones de distintos actores en diferentes contextos. Se realizó un análisis de los datos resaltados de las universidades, donde se muestra la relación de cada elemento de las categorías mencionadas.

Con lo anterior surge la elaboración de la red de las universidades y sus estrategias, con la finalidad de obtener medidas de grado nodal, núcleo y la partición de clusters para generar la interpretación apropiada del fenómeno.

\section{Resultados y discusión}

Se presentan resultados en los que se destacan estrategias claves que las IES trabajan hasta el momento en México, lo que permite a los estudiantes visualizar la oferta de cada una de las universidades. También se muestran la cantidad de materias que ofrece el plan de estudio de cada una de las instituciones y cada una de las consideraciones que les son útiles para el perfil de egreso de sus futuros clientes, con énfasis en aspectos de creatividad, investigación de mercados y estrategias de mercado, así como factores de diferenciación de mercadotecnia digital e innovación. Igualmente, se destaca que el clúster de universidades trabaja bajo un esquema de búsqueda de oportunidades, y apuestan a la dirección de las empresas como estrategia para la ofertar de sus planes.

A continuación, la tabla 1 muestra solo un resumen de las principales universidades del país de México y lo que buscan para sus estudiantes y posicionarse en el mercado. Y un análisis de los elementos y atributos que trabajan para la oferta de sus posgrados en el área de la mercadotecnia.

Tabla 1

Plan de estudios y perfil de egreso de las IES (Instituciones de Educación superior) de México.

\begin{tabular}{|c|c|l|}
\hline $\begin{array}{c}\text { Plan de } \\
\text { Estudios }\end{array}$ & Universidad & \multicolumn{1}{c|}{ Perfil de egreso } \\
\hline 18 & $\begin{array}{c}\text { Universidad } \\
\text { Popular Autónoma } \\
\text { del Estado de } \\
\text { Puebla }\end{array}$ & $\begin{array}{l}\text { Toma decisiones asertivas en el ámbito de la mercadotecnia analizando tendencias de } \\
\text { vanguardia. } \\
\text { Innova en método de análisis de decisiones para la investigación de mercados. } \\
\text { Diseña estrategias comerciales innovadoras a través de la sistematización del } \\
\text { conocimiento práctico sobre modelos de mercadotecnia. } \\
\text { Gestiona soluciones estratégicas con base en el pensamiento creativo e innovador } \\
\text { para comparar comportamientos de consumo y solucionar problemas en el campo de } \\
\text { la dirección y mercadotecnia. }\end{array}$ \\
\hline 14 & Universidad de & $\begin{array}{l}\text { Será un profesional competitivo, con capacidad analítica y habilidad inferir, que se } \\
\text { desempeña en el ámbito de las funciones y procesos del marketing, cuyos } \\
\text { conocimientos le permitirán desempeñarse de manera responsable y creativa en } \\
\text { puestos tanto funcionales como directivos. Poseerá un dominio en la implementación }\end{array}$ \\
\hline
\end{tabular}




\begin{tabular}{|c|c|c|}
\hline & & $\begin{array}{l}\text { de las estrategias comerciales, diseñadas con alto valor, basados en el conocimiento } \\
\text { continuo de los mercados de consumo y enmarcadas en los criterios de } \\
\text { sustentabilidad. }\end{array}$ \\
\hline N/A & $\begin{array}{c}\text { Universidad de } \\
\text { Guadalajara }\end{array}$ & $\begin{array}{l}\text { Será un profesional de alto nivel, líder en el ámbito de las funciones y procesos del } \\
\text { marketing, cuyos conocimientos le permitan desempeñarse con responsabilidad } \\
\text { social, creatividad y destreza en puestos directivos, especialmente en empresas } \\
\text { competitivas y organizaciones que aspiran al reconocimiento y valoración de la } \\
\text { sociedad. Capaz de buscar nuevas oportunidades en los mercados ya sea como } \\
\text { empleado o como emprendedor. } \\
\text { Amplio dominio en el diseño e implementación de las estrategias comerciales y } \\
\text { actividades que garantizan su desarrollo, aplicando con visión integral y con eficiencia } \\
\text { los medios y recursos. }\end{array}$ \\
\hline $\begin{array}{c}44 \\
\text { materias }\end{array}$ & Tec Milenio & $\begin{array}{l}\text { Implementarás efectivamente los procesos más relevantes de la mercadotecnia } \\
\text { digital. Identificar y analizar las diferentes áreas de oportunidad del entorno. Conocer } \\
\text { y manejar distintas herramientas mercadológicas con el propósito de satisfacer a sus } \\
\text { clientes, así mismo estar en posibilidades de proporcionar utilidad y valor a la } \\
\text { empresa. }\end{array}$ \\
\hline $\begin{array}{c}18 \\
\text { materias }\end{array}$ & $\begin{array}{l}\text { Universidad } \\
\text { Anáhuac México } \\
\text { Sur }\end{array}$ & $\begin{array}{l}\text { Permitirá identificar áreas de oportunidad, y lograr, con una visión de negocio clara y } \\
\text { objetiva, impulsar las ventas de una empresa o negocio, a través del reconocimiento } \\
\text { de la marca. }\end{array}$ \\
\hline N/A & $\begin{array}{l}\text { Universidad } \\
\text { Iberoamericana }\end{array}$ & $\begin{array}{l}\text { Líder interdisciplinario y creativo para desarrollar, dirigir y evaluar planes estratégicos } \\
\text { de mercadotecnia y actitudes para la creatividad e innovación en todas las áreas de la } \\
\text { mercadotecnia, para así crear estrategias y gestionar decisiones directivas }\end{array}$ \\
\hline $\begin{array}{c}16 \\
\text { materias }\end{array}$ & $\begin{array}{l}\text { Universidad } \\
\text { Iberoamericana }\end{array}$ & $\begin{array}{l}\text { Fundamentos y evolución de las técnicas, metodologías y modelos de mercadotecnia } \\
\text { y modelos de administración, control y dirección de áreas estratégicas de } \\
\text { mercadotecnia, entre otras. }\end{array}$ \\
\hline N/A & $\begin{array}{l}\text { Universidad de } \\
\text { Negocios ISEC }\end{array}$ & $\begin{array}{l}\text { Obtendrá conocimientos para detectar focos de negocios rentables para la empresa. } \\
\text { Identificará las necesidades de los clientes a través de la aplicación de investigación } \\
\text { de mercados y conocerá la evaluación financiera de las campañas de publicidad. }\end{array}$ \\
\hline $\begin{array}{c}17 \\
\text { materias }\end{array}$ & $\begin{array}{l}\text { Universidad } \\
\text { Autónoma del } \\
\text { Estado de México }\end{array}$ & $\begin{array}{l}\text { Faculta al estudiante para tener conocimiento a fondo del consumidor y de su } \\
\text { mercado, analizando con atención los factores de precio, producto, ámbito geográfico } \\
\text { y distribución, vinculándolo también de una manera estratégica, teniendo siempre en } \\
\text { mente la resolución de las necesidades sociales, de forma tal que la organización en } \\
\text { su conjunto se vea beneficiada }\end{array}$ \\
\hline $\begin{array}{c}18 \\
\text { materias }\end{array}$ & Universidad Ítaca & $\begin{array}{l}\text { La implementación de sistemas de administración de la operación con orientación al } \\
\text { mercado y el consumidor. La estructura de costos y toma de decisiones del precio, las } \\
\text { estrategias de comercialización y las técnicas utilizadas para el desarrollo y la correcta } \\
\text { implementación de un plan de mercadotecnia. }\end{array}$ \\
\hline $\begin{array}{c}15 \\
\text { materias }\end{array}$ & Universidad ICEL & $\begin{array}{l}\text { Planeará y dirigirá el área de Mercadotecnia, utilizando los métodos y técnicas } \\
\text { especializadas que le permitan desarrollar estrategias competitivas y tomar } \\
\text { decisiones para el desarrollo comercial de la empresa o institución y poseerá el } \\
\text { conocimiento sobre diversas áreas funcionales de la empresa o institución, como } \\
\text { dirección general, recursos humanos, finanzas, producción y comunicación } \\
\text { organizacional }\end{array}$ \\
\hline $\begin{array}{c}15 \\
\text { materias }\end{array}$ & $\begin{array}{c}\text { Universidad } \\
\text { Tecnológica de } \\
\text { México }\end{array}$ & $\begin{array}{l}\text { Podrá diseñar estrategias de mercadotecnia digital para diversos productos o } \\
\text { servicios. Será capaz de proponer mecanismos para la protección intelectual de las } \\
\text { soluciones y contenidos generados en un e-business y tendrá las herramientas para } \\
\text { diseñar campañas digitales de promoción y venta basadas en influencers y trending } \\
\text { topics. }\end{array}$ \\
\hline
\end{tabular}

Fuente: Elaboración propia con base en páginas web de las universidades.

En la segunda etapa, con relación a el análisis de redes sociales, se trabajo una métrica para entender a la red y sus actores (Instituciones de Educación Superior y factores), con un análisis del núcleo de datos seleccionados 
para correr en el programa Pajek, organizando los datos según el número de relaciones que impactan en la red de las universidades en México que ofertan programas de posgrado en el área de mercadotecnia con cuestiones afines. Con la finalidad de establecer cuantos vértices (número de líneas que se presentan en la red) están unidos a un núcleo y medir sus relaciones (factores seleccionados se destaca, en la medida numérica de las IES, la Universidad Popular Autónoma del Estado de México como número uno (con mayores relaciones), resultado 10 relaciones (vértices) en la red, entre ellas, toma de decisiones, tendencias y dirección; en segundo lugar se ubica la Universidad de Sonora, con 6 vértices impactando estrategias comerciales y dirección; como lugar 3 la Universidad de Guadalajara, con 5 vértices como responsabilidad social y dirección; y en 4 lugar la Universidad Tec Milenio, con relaciones en identificar áreas de oportunidad y mercadotecnia digital. La tabla 2, resume en que lugar se ubican todos los elementos de la red social según su número de relaciones en los factores seleccionados de la oferta de posgrados. Los números de la segunda columna muestra la significancia que tiene en la red cada factor, correspondiendo a las universidades el uno a la Universidad Popular Autónoma del Estado de México con mayores relaciones y el primer factor arrojado corresponde a toma decisiones con el número 12; estos corren en orden descendente. Se generan en ese orden, ya que la tabla fue arrojada por el sofware Pajek. Y la primera columna muestra el número de elementos estudiados en la red que corresponde a 23.

Tabla 2

Número de grados de la red.

Permutación de edición: 1 . numeración de Núcleo

+ grado de la nueva visualización de N1

\begin{tabular}{|c|c|c|}
\hline 1 & 19 & Estrategias comerciales \\
\hline 2 & 17 & Dirección \\
\hline 3 & 6 & Universidad Iberoamericana \\
\hline 4 & 2 & Universidad de Sonora \\
\hline 5 & 3 & Universidad de Guadalajara \\
\hline 6 & 10 & Universidad ICEL \\
\hline 7 & 15 & Creatividad \\
\hline 8 & 1 & Universidad Popular Autónoma del Estado de México \\
\hline 9 & 16 & Competitividad \\
\hline 10 & 12 & Toma de decisiones \\
\hline 11 & 7 & Universidad de Negocios ISEC \\
\hline 12 & 23 & Identificar áreas de oportunidad \\
\hline 13 & 18 & Responsabilidad \\
\hline 14 & 4 & Tec Milenio \\
\hline 15 & 20 & Mercadotecnia digital \\
\hline 16 & 22 & Investigación de mercados \\
\hline 17 & 14 & Innovación \\
\hline 18 & 5 & Universidad Anáhuac México Sur \\
\hline 19 & 8 & Universidad Autónoma del Estado de México \\
\hline 20 & 9 & Universidad Itaca \\
\hline 21 & 21 & Impulsar ventas \\
\hline 22 & 11 & Universidad Tecnológica de México \\
\hline 23 & 13 & Tendencias \\
\hline
\end{tabular}

Fuente: Elaboración propia (2021)

La red de la figura 1 se compone de 12 nodos que representan a los elementos que trabajan las universidades en México y lo que se destaca, aunque también se consideran los valores de la tabla 2 y los atributos 
seleccionados para el estudio, que prevalecen en las IES, destacando entre ellos estrategias comerciales, identificación de áreas de oportunidad y dirección de empresas.

Figura 1

Red o modelo matemático que muestra atributos de perfil de egreso en las IES en México.

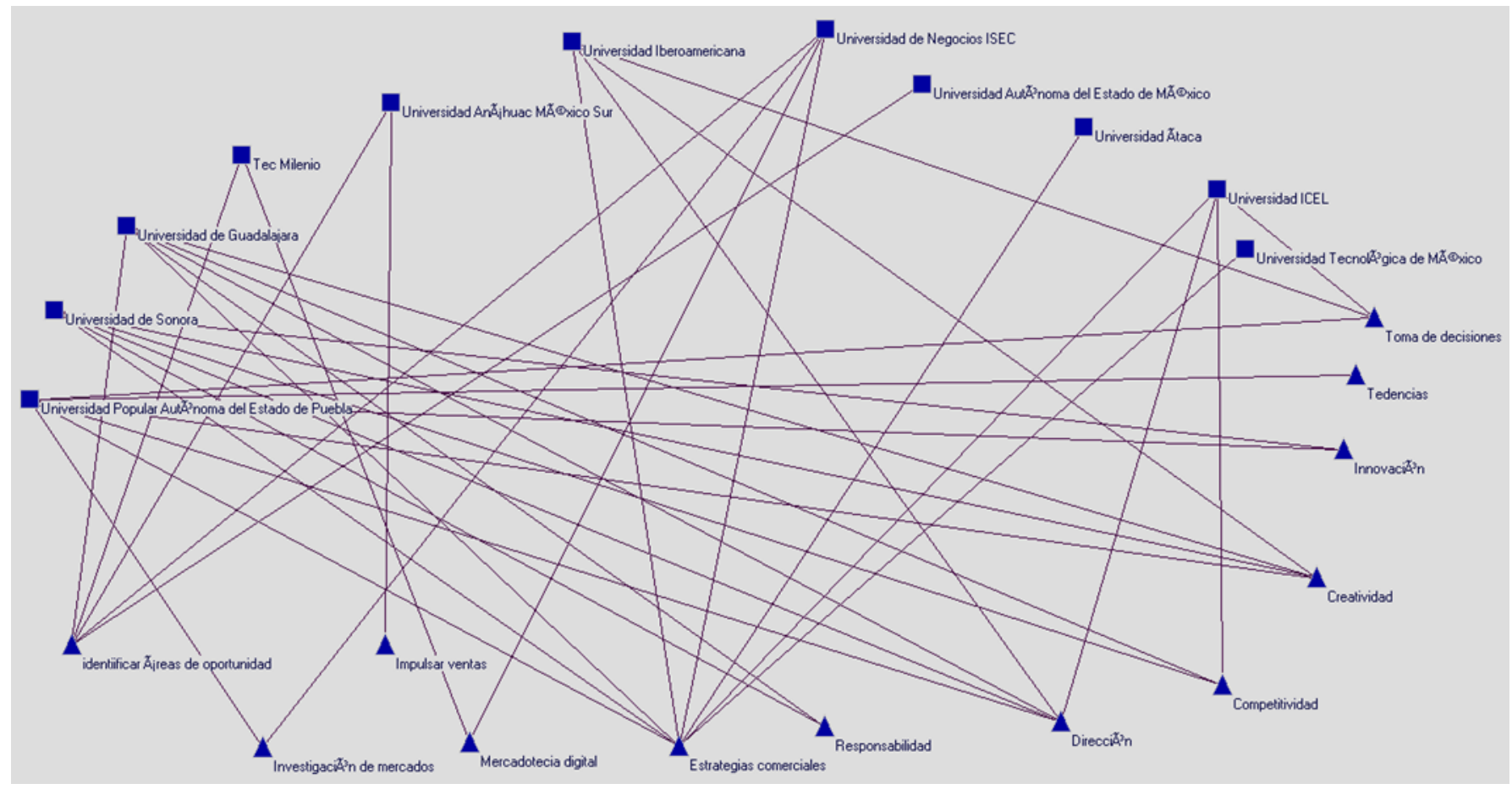

Fuente: Elaboración propia (2021)

Las redes permiten una compresión de la dinámica de los factores que impactan en los estudiantes para elegir un posgrado, lo que resulta fundamental para las IES que deseen abrir nuevos programas en el área de mercadotecnia y las actuales para desarrollar estrategias de mejora con el objetivo de lograr un posicionamiento más exitoso y una comprensión de elementos que pueden impactar en los distintos mercados, en las dinámicas actuales y según las necesidades de las empresas con los factores encontrados.

Como segunda corrida de la red de atributos mencionados anteriormente se decidió hacer el análisis de clusteres para determinar como se clasifican los factores y su comportamiento arrojando como resultado dos grupos, el primero (c1) con once relaciones y el segundo (c2) presento el mismo número de relaciones; en el cluster uno destaca la identificacion de áreas de oportunidad, impulsar las ventas, mercadotecnia digital, innovación, creatividad, responsabilidad y estrategias comerciales y en el cluster dos se destacan competitividad, dirección e investigación de mercado como lo muestra la figura 2. 
Figura 2

Red o modelo matemático de clusteres que muestra atributos de perfil de egreso en las IES en México

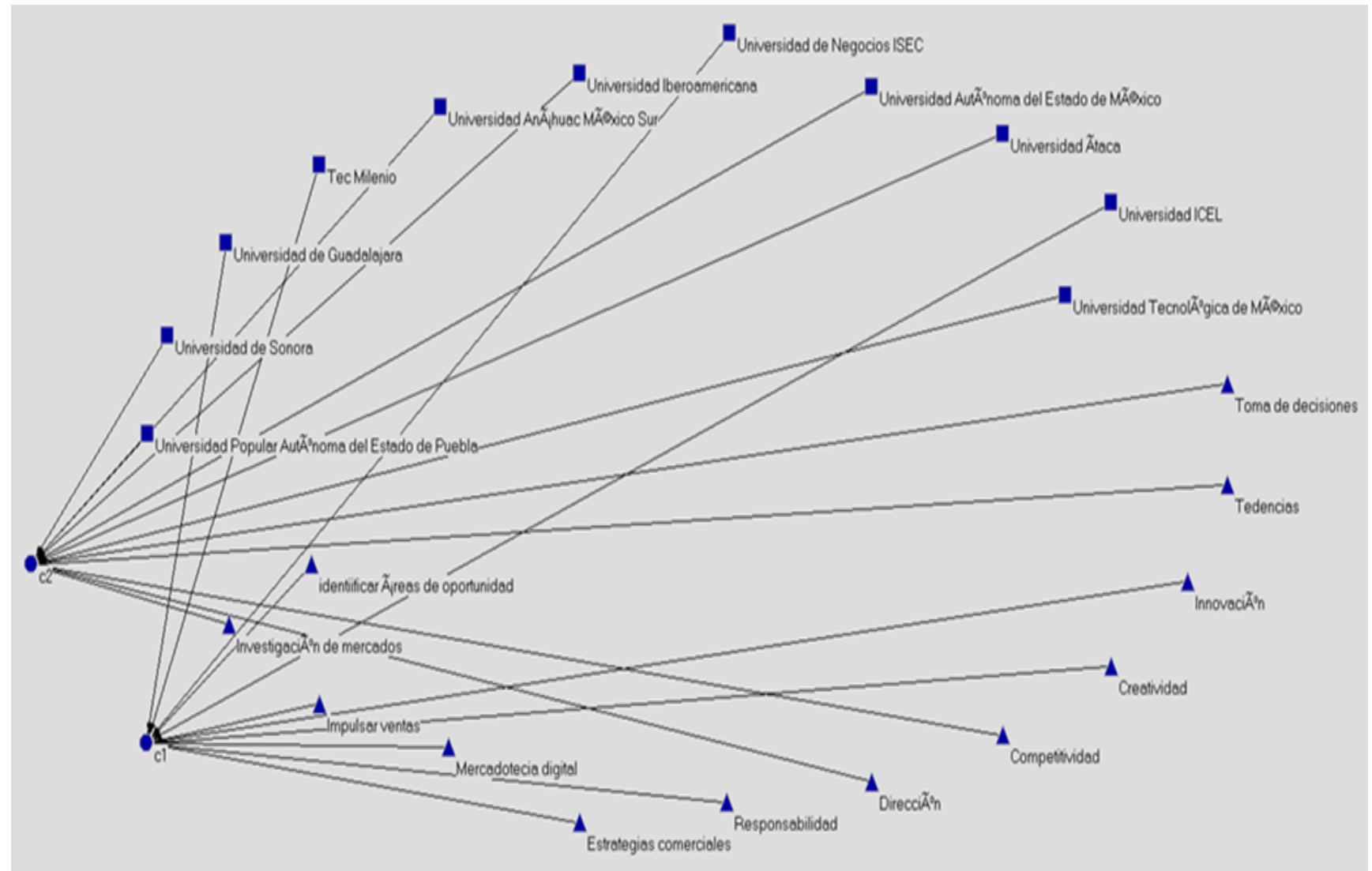

Fuente: Elaboración propia (2021)

En relación a un tercer análisis en el sofware Pajek, se genero una segunda red, para determinar el impacto en organización de factores en dos grupos (clusters), de acuerdo a relaciones de significancia como lo muestra la tabla 3, donde la columna de (Val) corresponde al grupo (cluster) formado por factores y universidades; y vertex (vértices) es el número de líneas (relaciones) que se generaron de acuerdo al análisis de redes de lo que ofertan las IES. Donde el grupo dos se conformó por 7 universidades (Universidad Popular Autónoma del Estado de Puebla, Universidad de Sonora, Universidad Anáhuac de México, Universidad Iberoamericana, Universidad Autónoma del Estado de México, Univerisidad de Negocios ICEL) y cinco atributos entre ellos, toma de decisiones y tendencias. Para el cluster uno resultaron cuatro universidades (Universidad de Guadalajara, Universidad Tec Milenio, Universidad Itaca, Universidad Universidad ICEL con atributos como innovación y estrategias comerciales, resultando con más significancia el grupo 2 en relación a los vértices que lo conforman que son mayores que los del grupo uno. 
Tabla 3

Número de grados de la red

Partición de edición: 1. Partición aleatoria en 2 grupos (23)

\begin{tabular}{|c|c|c|}
\hline Vertex & Val & Label \\
\hline 1 & 2 & Universidad Popular Autónoma del Estado de Puebla \\
\hline 2 & 2 & Universidad de Sonora \\
\hline 3 & 1 & Universidad de Guadalajara \\
\hline 4 & 1 & Universidad Tec Milenio \\
\hline 5 & 2 & Universidad Anáhuac de México \\
\hline 6 & 2 & Universidad Iberoamericana \\
\hline 7 & 1 & Universidad de Negocios ISEC \\
\hline 8 & 2 & Universidad Autónoma del Estado de México \\
\hline 9 & 2 & Universidad Itaca \\
\hline 10 & 1 & Universidad ICEL \\
\hline 11 & 2 & Universidad Tecnológica de México \\
\hline 12 & 2 & Toma de decisiones \\
\hline 13 & 2 & Tendencias \\
\hline 14 & 1 & Innovación \\
\hline 15 & 1 & Creatividad \\
\hline 16 & 2 & Competitividad \\
\hline 17 & 2 & Dirección \\
\hline 18 & 1 & Responsabilidad social \\
\hline 19 & 1 & Estrategias comerciales \\
\hline 20 & 1 & Mercadotecnia digital \\
\hline 21 & 1 & Impulsar la ventas \\
\hline 22 & 2 & Investigación de mercados \\
\hline 23 & 1 & Identificar áreas de oportunidades \\
\hline
\end{tabular}

Fuente: Elaboración propia (2021)

Las asociaciones que refleja la red se pueden cuantificar y vincular al desarrollo educativo y profesional de las universidades, las cuales pueden brindar múltiples beneficios en la elaboración y modificación de programas con la propuesta de factores encontrados, encaminados a una planeación estratégica y a la búsqueda actuales en el país para brindar valor a sus posgrados.

\section{Conclusiones}

Las tendencias actuales obligan a las universidades a buscar estrategias competitivas para su posicionamiento y desarrollo exitoso que lleve a objetivos claros y permita la ampliación de factores claves para cubrir las necesidades actuales en el entorno educativo y empresarial de diversos entornos.

Esta investigación mostró desde una perspectiva diferente, mediante un análisis de redes, observar como se comportan en la actualidad las tendencias y visualizar sus diversas relaciones a través de métricas, con el objetivo de que las IES analizen los requerimientos actuales y la pertinencia de sus posgrados.

Conviene resaltar la toma de decisiones, las estrategias comerciales y la identificación de oportunidades para ampliar la oferta educativa en las IES y trabajar la innovación mediante la potencialización de factores identificados en la revisión de la amplia gama de posgrados. Asimismo, surge la necesidad de que las universidades adopten la innovación y las estrategias idóneas para su gestión y aspectos relacionados con la integración de dirección en mercadotecnia como elementos fundamentales, para poder no solo competir sino 
también destacar en el mercado. Ya que la actualidad la educación tiene más exigencias, se requieren nuevas metodologías y trabajo en cada uno de sus programas y sus procesos educativos.

Y como lo menciona Ruiz, Gómez \& Cortes (2018) "en este proceso se deben difundir aspectos como la calidad, responsabilidad social, perfil de consumidores, competitividad de la planta docente, infraestructura y planes estratégicos por citar algunos componentes" (p.65)

Es necesario también que se considere la investigación de mercados y temas relacionados a las ventas, sumado a lo que ya se mencionó anteriormente para poder destacar en las distintas regiones, con la idea de desarrollar sus programas y promoverlos, así como transformar propuestas para promover una formación integral y completa para diferentes necesidades y demandas en distintos ámbitos.

Por otra parte, la evolución de los programas educativos ha crecido y actualmente existen universidades interesadas en ofertar nuevos programas para extender su oferta y diversificarse, lo que involucra una combinación de elementos estratégicos, pero que de diversas maneras puede llevar las IES a un crecimiento y desarrollo exitoso, asi como obtener y mejorar resultados.

Cada región es distinta y cada persona también, por tanto, los factores demandados no serán los mismos; estos dependerán de cada ámbito y de las necesidades que buscan satisfacer los clientes. El objetivo de la red es examinar un mapa a través del cual se puedan visualizar factores claves en las universidades para el desarrollo de sus programa y se puede construir un plan educativo dinámico y establecer estrategias para elevar la competitividad y los factores de éxito.

De la reflexión planteada en el párrafo anterior, se podrían identificar tendencias actuales y nuevas disciplinas derivadas del marketing y su influencia sobre las distintas áreas de la empresa y de la sociedad y destacar cómo lo identifica Alonso (2017) de entre todas ellas, nuevas tendencias globales dentro del ámbito empresarial como: digital marketing, neuromarketing y personal branding y Nieto, Ortega \& Turrent (2017) suman a estas las tendencias demográficas, tendencias socioculturales, tendencias tecnológicas, tendencias educativas y tendencias económicas.

\section{Referencias}

Alonso-González, A. (2017). Nuevas Tendencias del Marketing en las Ciencias Económicas y Administrativas. Cuadernos Latinoamericanos de administración, 13(25), 5-8.

Bermeo-Giraldo, M.., Correa, Y., Moya, L., Arias, M.., \& Arango-Botero, D. (2020). Evolución y tendencias investigativas sobre estrategias de gestión de conocimiento en instituciones de educación superior. Revista Virtual Universidad Católica del Norte, (60), 202-227.

Betancur, X. M., \& Murcia, J. A. (2019). El Análisis de Redes Sociales-ARS-como recursos metodológico para el estudio formal de redes de políticas públicas. Espacio Abierto, 28(3), 109-126.

Cueto, C., Peña, M., Reyes, P., \& Cobos, O. (2018). Resultados Preliminares para ofertar Posgrados de Calidad en la Universidad Autónoma de Nayarit. Unidad Académica Bahía de Banderas. EDUCATECONCIENCIA, 20(21), 268-290.

Hernández, R., \& López, T. (2011). Estudio de necesidades de capital humano con posgrado en administración en las medianas y grandes empresas. Revista Ciencia Administrativa, 36-45.

Luque, F., Lozano, L., \& Quiroz, A. (2017). Las Tendencias del Marketing: Cuales son y definiciones. RECIMUNDO: Revista Científica de la Investigación y el Conocimiento, 1(5), 974-988. 
Moral, G., VILLEGAS, V., Landero, J., \& Cornelio-Marí, E. (2020). Prospectiva: vertientes, tendencias y su aplicación en el ámbito socioeducativo. Ecos sociales, 8(23).

Muñoz, N., Avilés, C., Divicino, A., \& Ramirez, T. (2020). Competencias profesionales 2020 para la formación del posgrado en Comunicación Estratégica y Relaciones Públicas. Estudio de caso de la Universidad Autónoma de Guerrero. Controversias y Concurrencias Latinoamericanas, 12(21), 347-368.

Nieto, E.., Ortega M.,\& Turrent, G. (2017). Diseño curricular de un nuevo posgrado de negocios con un enfoque de innovación, competitividad, responsabilidad social y ética empresarial. Hitos de Ciencias Económico Administrativas, 23(67), 375-395.

Pombosa, E., Espín, E., Morales, N., Moyano, H., \& Burgos, R. (2018). Formación dual universitaria en Ecuador. Atlante Cuadernos de Educación y Desarrollo.

Rodríguez, M.., Pineda, D., \& Castro, C. (2020). Tendencias del marketing moderno, una revisión teórica. Revista ESPACIOS. ISSN, 798, 1015.

Ruiz, K., Gómez, M.., \& Cortes, J. (2018). Marketing de universidades, ¿̇oferta educativa o imagen?. Hitos de Ciencias Económico Administrativas, 24(68), 56-67.

Vega, J., González, M., González, A., \& Hernández, I. (2018). Análisis de la oferta educativa superior de pregrados universitarios de marketing en Colombia, en 2017. Cuadernos Latinoamericanos de Administración, 14(26).

Vélez, V., \& Sera, R. (2018). Rediseño curricular de la carrera de mercadotecnia desde una perspectiva intercultural: algunos apuntes. Revista Cognosis. ISSN 2588-0578, 3(2), 27-34.

Esta obra está bajo una Licencia Creative Commons

Attribución-NoCommercial 4.0 International

(cc) EY-NG 